OPEN ACCESS

Edited by:

Peter Schemmer,

Medical University of Graz, Austria

Reviewed by:

Bassem Soliman Hegab,

National Liver Institute, Egypt

Cristiano Quintini,

Cleveland Clinic, United States

*Correspondence:

Xiaoshun $\mathrm{He}$

gdtrc@163.com

Maogen Chen

chenmg3@mail.sysu.edu.cn

Weiqiang Ju

weiqiangju@163.com

tThese authors have contributed equally to this work

Specialty section: This article was submitted to

Visceral Surgery,

a section of the journal

Frontiers in Surgery

Received: 05 December 2020

Accepted: 15 April 2021

Published: 24 May 2021

Citation:

Chen $Z$, Hong $X$, Huang S, Wang $T$, Ma Y, Guo Y, Huang C, Zhao Q,

Guo Z, HeX, Ju W and Chen M (2021) Continuous Normothermic Machine Perfusion for Renovation of

Extended Criteria Donor Livers

Without Recooling in Liver Transplantation: A Pilot Experience.

Front. Surg. 8:638090.

doi: 10.3389/fsurg.2021.638090

\section{Continuous Normothermic Machine Perfusion for Renovation of Extended Criteria Donor Livers Without Recooling in Liver Transplantation: A Pilot Experience}

\author{
Zhitao Chen ${ }^{1,2,3+}$, Xitao Hong ${ }^{1,2,3+}$, Shanzhou Huang ${ }^{4}$, Tielong Wang ${ }^{1,2,3}$, Yihao Ma ${ }^{1,2,3}$, \\ Yiwen Guo ${ }^{1,2,3}$, Changjun Huang ${ }^{1,2,3}$, Qiang Zhao ${ }^{1,2,3}$, Zhiyong Guo ${ }^{1,2,3}$, Xiaoshun $\mathrm{He}^{1,2,3 *}$, \\ Weiqiang $\mathrm{Ju}^{1,2,3 *}$ and Maogen Chen ${ }^{1,2,3 *}$ \\ 1 Organ Transplant Center, First Affiliated Hospital of Sun Yat-Sen University, Guangzhou, China, ${ }^{2}$ Guangdong Provincial Key \\ Laboratory of Organ Donation and Transplant Immunology, Guangzhou, China, ${ }^{3}$ Guangdong Provincial International \\ Cooperation Base of Science and Technology (Organ Transplantation), Guangzhou, China, ${ }^{4}$ Department of General Surgery, \\ Guangdong Provincial People's Hospital, Guangdong Academy of Medical Sciences, School of Medicine, South China \\ University of Technology, Guangzhou, China
}

Background: Ischemia injury affects the recovery of liver allograft function. We propose a novel technique aimed at avoiding a second ischemic injury: transplanting an extended criteria donor (ECD) liver directly under normothermic machine perfusion (NMP) without recooling. We studied two cases to evaluate the efficacy and safety of this technique.

Methods: The perioperative characteristics and postoperative outcomes of two recipients of ECD livers were analyzed. Both transplantations were performed with continuous normothermic machine perfusion without recooling.

Result: In case 1, the cause of donor death was anoxia, and the donor liver had hypernatremia before procurement. The recipient was diagnosed with decompensated cirrhosis. His model for end-stage liver disease (MELD) score was 38. In case 2, the donor liver was from a donor after cardiac death (DCD), and the donor had elevated aspartate aminotransferase (AST) and alanine aminotransferase (ALT) levels. The recipient was diagnosed with acute hepatic failure. His MELD score was 35. Both donor livers were maintained under NMP and then transplanted without recooling. The peak ALT and AST levels after surgery were 452 and $770 \mathrm{U} / \mathrm{L}$ in case 1 and 100 and $592 \mathrm{U} / \mathrm{L}$ in case 2. Neither early allograft dysfunction (EAD) nor primary graft non-function (PNF) was present in these two cases.

Conclusion: In conclusion, our results demonstrate that continuous NMP without recooling is efficacious and safe for LT with extended criteria donor livers. Further investigations of this technique will be performed to confirm these promising results.

Keywords: continuous normothermic machine perfusion, extended criteria donor liver, early allograft dysfunction, donor after cardiac death, allograft 


\section{INTRODUCTION}

Liver transplantation (LT) is a successful treatment option for end-stage liver disease (ESLD) of various etiologies (1). The 1 -year survival rate after LT is $\sim 90 \%$ thanks to tremendous improvements in surgical methods and immunosuppression (2). Ischemia-reperfusion injury (IRI) constitutes an important cause of PNF, EAD, and biliary complications after LT (3). Normothermic machine perfusion (NMP) is an organ preservation technique that entails continuous or pulsatile circulation of perfusate through the graft and has proven effective in ameliorating IRI (4). In conventional NMP, the liver graft is usually flushed with cold solution before implantation, and IRI is still inevitable (5). We propose a novel technique aimed at avoiding a second ischemic injury by transplanting ECD livers directly under NMP without recooling. We have reported on one patient who underwent this technique, who showed good recovery (6). In this study, we report another two cases to further evaluate the efficacy and safety of this technique.

\section{MATERIALS AND METHODS}

All the procedures were performed in accordance with the ethical standards of the responsible committee on human institutional and national experimentation and with the Helsinki Declaration of 1964 and its later revisions.

Two patients who had undergone OLT with continuous NMP without recooling were included in the study. The recipient pre-transplantation data and the perioperative data, including the perfusion parameters and postoperative complications, were assessed and analyzed.

\section{Description of Continuous NMP Without Recooling and Implantation}

Details about this technique have been described in previously published articles. Briefly, the donor liver was procured under a standard perfusion technique, and the organ was preserved in a University of Wisconsin (UW) solution prior to perfusion. Afterwards, the donor liver was reprocessed in NMP to $37^{\circ} \mathrm{C}$ following the flush of lactated Ringer's solution. After ligation of the cystic duct, a tube was placed in it to drain bile. The cannula was placed separately into the infrahepatic inferior vena cava (IHIVC), portal vein (PV) via an interposition vascular graft (the donor iliac artery), and the gastroduodenal artery (GDA), respectively, then connected to a liver assist device for perfusion. The perfusate is described in Table 1. Electrolyte balance and acid-base equilibrium were adjusted and maintained throughout

Abbreviations: AKI, acute kidney injury; AST, aspartate transaminase; ALT, alanine transaminase; DBD, donor after brain death; DCD, donor after cardiac death; EAD, early allograft dysfunction; ECD, extended criteria donor; ESLD, end-stage liver disease; GDA, gastroduodenal artery; HBV, hepatitis B virus; HA, hepatic artery; ICU, intensive care unit; IRI, ischemia-reperfusion injury; INR, international normalized ratio; IHIVC, infrahepatic inferior vena cava; LT, liver transplantation; MELD, model for end-stage liver disease; NMP, normothermic machine perfusion; PV, portal vein; PNF, primary graft non-function; POD, postoperative day; RCT, randomized clinical trials; SHIVC, suprahepatic inferior vena cava; Tbil, total bilirubin; UW, University of Wisconsin.
TABLE 1 | The components of the perfusate solution.

\begin{tabular}{ll}
\hline Components & \\
\hline Crossed-matched leucocyte-depleted washed red cells Gelofusine ${ }^{\circledR}$ & $8 \mathrm{U}$ \\
$5 \%$ sodium bicarbonate & $800 \mathrm{ml}$ \\
Heparin & $150 \mathrm{ml}$ \\
$10 \%$ calcium gluconate & $37,500 \mathrm{U}$ \\
$25 \%$ magnesium sulfate & $30 \mathrm{ml}$ \\
Methylprednisolone & $2 \mathrm{ml}$ \\
Compound Amino Acid Injection & $500 \mathrm{mg}$ \\
Imipenem cilastatin & $250 \mathrm{ml}$ \\
Metronidazole & $0.5 \mathrm{~g}$ \\
\end{tabular}

the perfusion process. Perfusion was continuous from the time of preservation to the reperfusion.

After dissection of the diseased liver, the donor liver was transplanted with an undergoing NMP circuit. We first anastomosed the donor suprahepatic inferior vena cava (SHIVC) and the recipient counterpart in an end-to-end fashion. Subsequently, PV and HA were anastomosed with the donor's counterpart in an end-to-end fashion. During the anastomosis, the blood supply was not stopped. Afterwards, the clamps on the recipient's PV and HA were removed to restore the blood supply to the donor liver soon after NMP was stopped, after removal of the HA and PV cannula (Figure 1). The IHIVC cannula was then removed, and the IHIVC was anastomosed in an end-toend fashion. After removal of the drainage tube, the common bile duct was anastomosed end-to-end.

\section{Posttransplant Care}

After the transplantation, all patients were transferred to the intensive care unit with the endotracheal tube. After the tubes were removed, the patients were transferred to the general ward for further care. Biomedical values including regular blood test, transaminase, and bilirubin were tested and documented every day in the first week. Routine Doppler ultrasound of the liver graft blood flow and biliary tract was performed once every 2 days for 7 days. The immunosuppressive regimen was similar for all patients and included tacrolimus and mycophenolate mofetil. Basiliximab $(20 \mathrm{mg}$ ) was administered intraoperatively before closing the abdomen and the 4 th day postoperation by intravenous pump for induction immunotherapy. The blood levels of tacrolimus were $8-12 \mathrm{ng} / \mathrm{ml}$ in the first month, followed by $4-8 \mathrm{ng} / \mathrm{ml}$ thereafter.

\section{RESULTS}

Data of these two cases are presented in Table 2. In case 1, the serum AST, ALT, and total bilirubin (Tbil) levels of the donor before procurement were $56.8 \mathrm{U} / \mathrm{L}, 26.2 \mathrm{U} / \mathrm{L}$, and $10.7 \mu \mathrm{mol} / \mathrm{L}$, respectively. Hypernatremia (sodium, $165 \mathrm{mmol} / \mathrm{L}$ ), anoxia as the cause of death, and a long cold ischemia time of more than $8 \mathrm{~h}$ were the risk factors for failed liver transplantation. In case 2, the AST, ALT, and TBil levels of the donor were $163 \mathrm{U} / \mathrm{L}, 151$ 
A

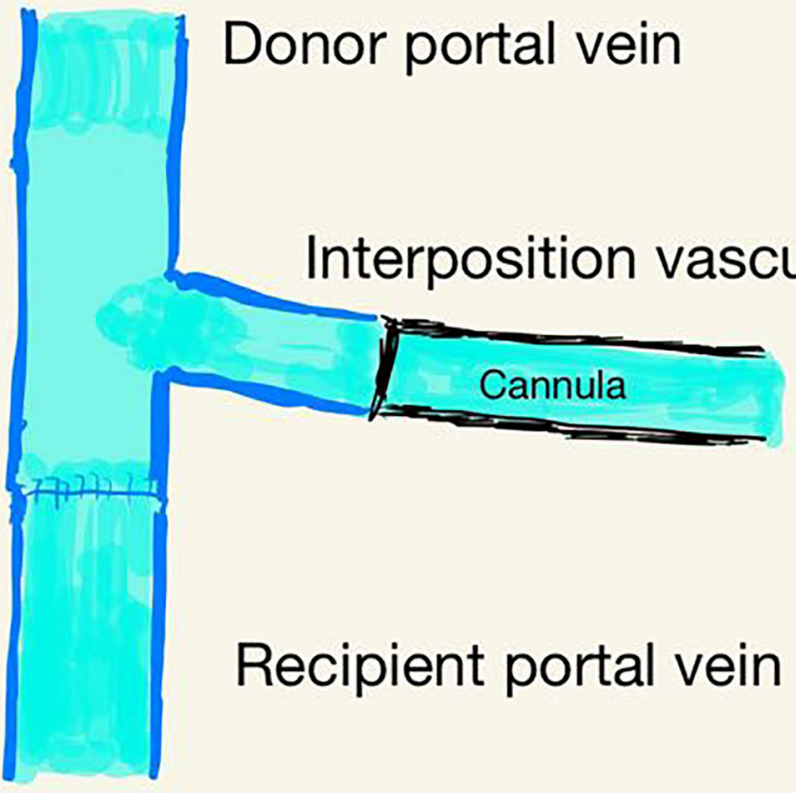

B

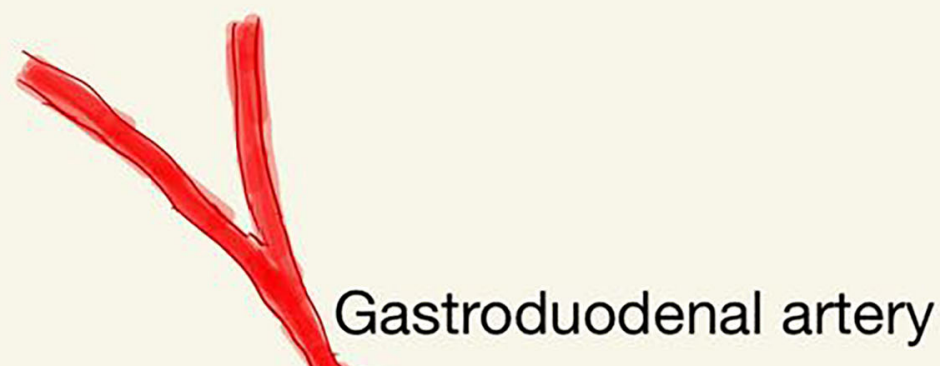

Donor common hepatic artery

\section{Recipient hepatic artery}

FIGURE 1 | Schematic diagram of portal vein (A) and hepatic artery (B) anastomosis.

$\mathrm{U} / \mathrm{L}$, and $6.2 \mu \mathrm{mol} / \mathrm{L}$, respectively. Risk factors were as follows: donor after cardiac death (DCD), anoxia as the cause of death, and a cold ischemia time of more than $10 \mathrm{~h}$. All risk factors made these two donors ECDs.
The recipient patient in case 1 was diagnosed with hepatitis $\mathrm{B}$ virus-associated (HBV) decompensated cirrhosis. He had a history of esophageal and gastric variceal bleeding and abdominal bleeding. His serum AST, ALT, and Tbil levels were $11 \mathrm{U} / \mathrm{L}$, 
TABLE 2 | Perioperative data of 2 patients with continuous NMP without re-cooling.

\begin{tabular}{|c|c|c|}
\hline & Donor 1 & Donor 2 \\
\hline Donor type & DBD & DCD \\
\hline Sodium (mmol/L) & 165 & 150 \\
\hline Potassium (mmol/L) & 3.66 & 3.50 \\
\hline Hemoglobin (g/L) & 114 & 79 \\
\hline AST(U/L) & 56.8 & 163 \\
\hline $\mathrm{ALT}(\mathrm{U} / \mathrm{L})$ & 26.2 & 151 \\
\hline GGT(U/L) & 27 & 31 \\
\hline Bilirubin (mmol/L) & 10.7 & 6.2 \\
\hline Anhepatic time, min & 86 & 51 \\
\hline CIT, h & 8 & 10 \\
\hline \multirow[t]{2}{*}{ WIT, min } & 0 & 8 \\
\hline & Patient 1 & Patient 2 \\
\hline MELD & 38 & 35 \\
\hline Preoperative AST(U/L) & 11 & 50 \\
\hline Preoperative ALT(U/L) & 39 & 35 \\
\hline Preoperative Bilirubin (mmol/L) & 152.8 & 440.8 \\
\hline Total operation time (mins) & 470 & 460 \\
\hline Anhepatic time (mins) & 55 & 51 \\
\hline Intraoperative transfusions $(U)$ & 26 & 20 \\
\hline Blood loss (ml) & 6,000 & 4,000 \\
\hline ICU length-of-stay (h) & 105 & 216 \\
\hline Peak AST(U/L) & 770 & 592 \\
\hline Peak ALT(U/L) & 452 & 100 \\
\hline INR on POD 7 & 1.35 & 1.23 \\
\hline Bilirubin (mmol/L) on POD7 & 86.8 & 78.9 \\
\hline EAD & 0 & 0 \\
\hline PNF & 0 & 0 \\
\hline Biliary anastomotic strictures & 0 & 0 \\
\hline Hepatic artery complications & 0 & 0 \\
\hline Acute kidney injury & 0 & 1 \\
\hline
\end{tabular}

DBD, Donor after brain death; DCD, Donor after cardiac death; AST, Aspartate aminotransferase; ALT, Alanine aminotransferase; GGT, $\gamma$-glutamyl transpeptidase; CIT, Cold ischemia time; WIT, Warm ischemia time; MELD, Model for end-stage liver diseases; ICU, Intensive care unit; INR, International normalized ratios; EAD, Early allograft dysfunction; PNF, Primary nonfunction; POD, postoperative day.

$39 \mathrm{U} / \mathrm{L}$, and $152.8 \mu \mathrm{mol} / \mathrm{L}$, respectively. His MELD score was 38. The recipient in case 2 was diagnosed with acute hepatic failure and hepatic encephalopathy. He had a history of cerebral infarction, hypertension, and HBV infection. His AST, ALT, and Tbil levels were $50 \mathrm{U} / \mathrm{L}, 35 \mathrm{U} / \mathrm{L}$, and $440.8 \mu \mathrm{mol} / \mathrm{L}$, respectively. His MELD score was 38.

NMP was performed for a total of $7 \mathrm{~h}$ for each donor liver (Figure 2). During the perfusion process, the electrolyte balance and acid-base equilibrium were adjusted and maintained. The $\mathrm{pH}$ during perfusion was adjusted and maintained at 7.37 and 7.42 in cases 1 and 2, respectively. The lactate concentration dropped significantly, from 9.9 to $2.4 \mathrm{mmol} / \mathrm{L}$ after $80 \mathrm{~min}$ of perfusion in case 1 and from 9.2 to $1.4 \mathrm{mmol} / \mathrm{L}$ after $80 \mathrm{~min}$ of perfusion in case 2 . The concentrations of calcium and sodium remained stable in both cases. The anhepatic time was 55 and $51 \mathrm{~min}$ in cases 1 and 2, respectively.

Postoperative data are presented in Table 2. The intensive care unit (ICU) stay times were 105 and $216 \mathrm{~h}$, respectively. The maximal ALT and AST were 452 and $770 \mathrm{U} / \mathrm{L}$ in case 1 and 100 and $592 \mathrm{U} / \mathrm{L}$ in case 2 , respectively. The international normalized ratio (INR) and TBil on postoperative day (POD) 7 were 1.35 and $86.8 \mathrm{mmol} / \mathrm{L}$ in case 1 , respectively, and 1.23 and $78.9 \mathrm{mmol} / \mathrm{L}$ in case 2 (Figure 3). Neither EAD nor PNF was presented in either case. Patient 2 suffered from acute kidney injury (AKI).

\section{DISCUSSION}

In conventional LT, cryopreservation can reduce the physiological metabolism of cells but cannot slow down the damage to cell integrity (7). IRI represents one of the major challenges during liver surgery and LT. To minimize the risk of PNF or EAD caused by IRI, we designed a novel method called continuous NMP without recooling for ECD livers. A case report on the feasibility of avoiding cooling was authored by Fabrizio et al. (8). However, they do not describe the details of the specific procedures. Ju et al. were the first to describe how to avoid a second cooling before implantation and proved that doing so helps reduce the risk of PNF or EAD (6). In this study, we report two cases of LT following this technique.

ECD livers are thought to be of lower-than-average quality and to be associated with poorer postoperative outcomes (9). Maring et al. suggested that ECD livers are associated with older donor age, longer warm ischemia time, moderate or severe microvascular steatosis, and HBsAg positivity (10). The review by Vodkin et al. noted that ECD livers are correlated with older donor age, steatosis, DCD, and donors with an increased risk of disease transmission (11). In our study, both donor livers had several risk factors, confirming that they were ECD livers. They were not suited for transplantation with conventional cold preservation. Tchilikidi et al. suggested that ECD transplantation required measures for graft preservation and assessment (12). NMP provides an effective method for salvaging ECD organs. Davide et al. retrospectively studied 34 transplantations of DCD donors using NMP and showed that it would be feasible for DCD LT (13). The prospective clinical trial of Otto et al. reported that NMP was a safe option for risky donor livers and increased the number of available donor livers (14). Nevertheless, current NMP techniques have potential problems and need innovation. Our novel technique is designed to solve this problem: during preservation and implantation, the blood supply is not stopped. In our cases, the laboratory test results fell from above the normal ranges to within the normal ranges within 1 week. Neither EAD nor PNF was presented. However, further randomized clinical trial (RCT) data are needed to confirm its safety and efficacy.

The limitations of our study are as follows. First, the preservation (the establishment of portal interposition vascular graft and the connection of cannulas) and the implantation are complex and need to be further modified. In addition, its effectiveness needs to be proven in a RCT. 
A

pH

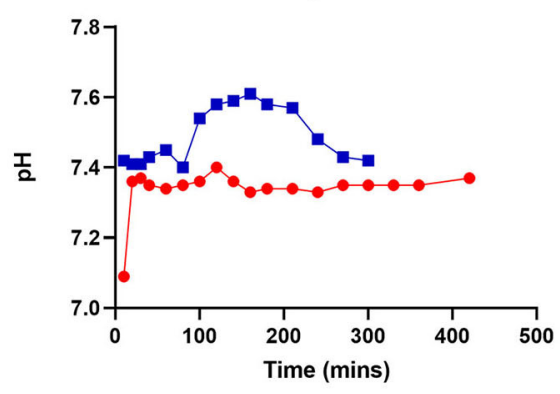

c

Patient 1

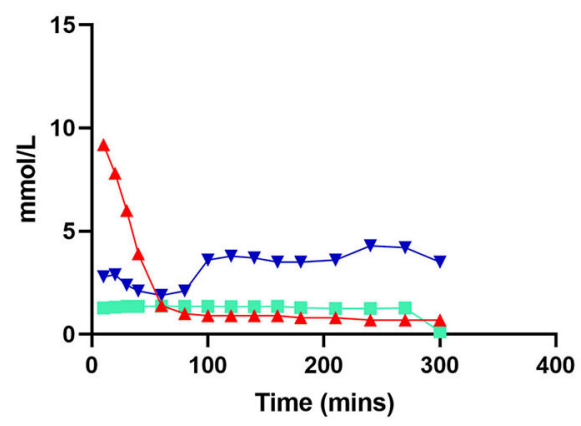

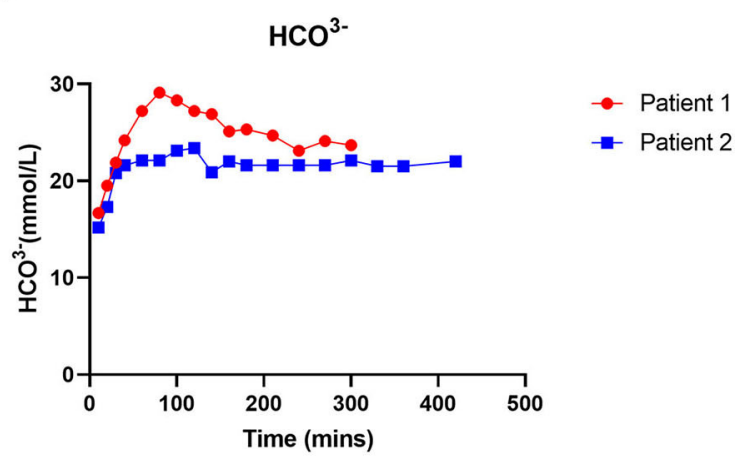

D

\section{Patient 2}

$$
\begin{aligned}
& \rightarrow \mathrm{Lac} \\
& \rightarrow \mathrm{K}^{+} \\
& -\mathrm{Ca}^{2+}
\end{aligned}
$$

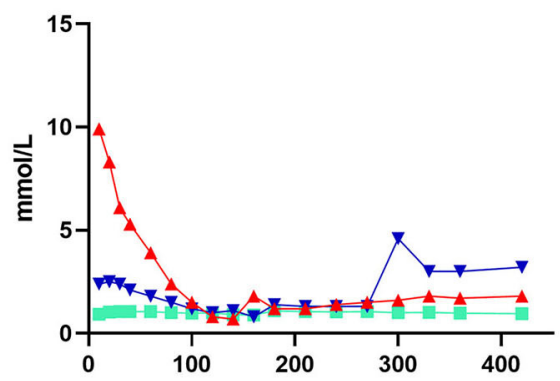

FIGURE 2 | Dynamic changes in physicochemical indexes during perfusion. (A) Changes in pH. (B) Changes in $\mathrm{HCO}^{3-}$. (C) Changes in lactate and ion concentrations in patient 1. (D) Changes in lactate and ion concentrations in patient 2.
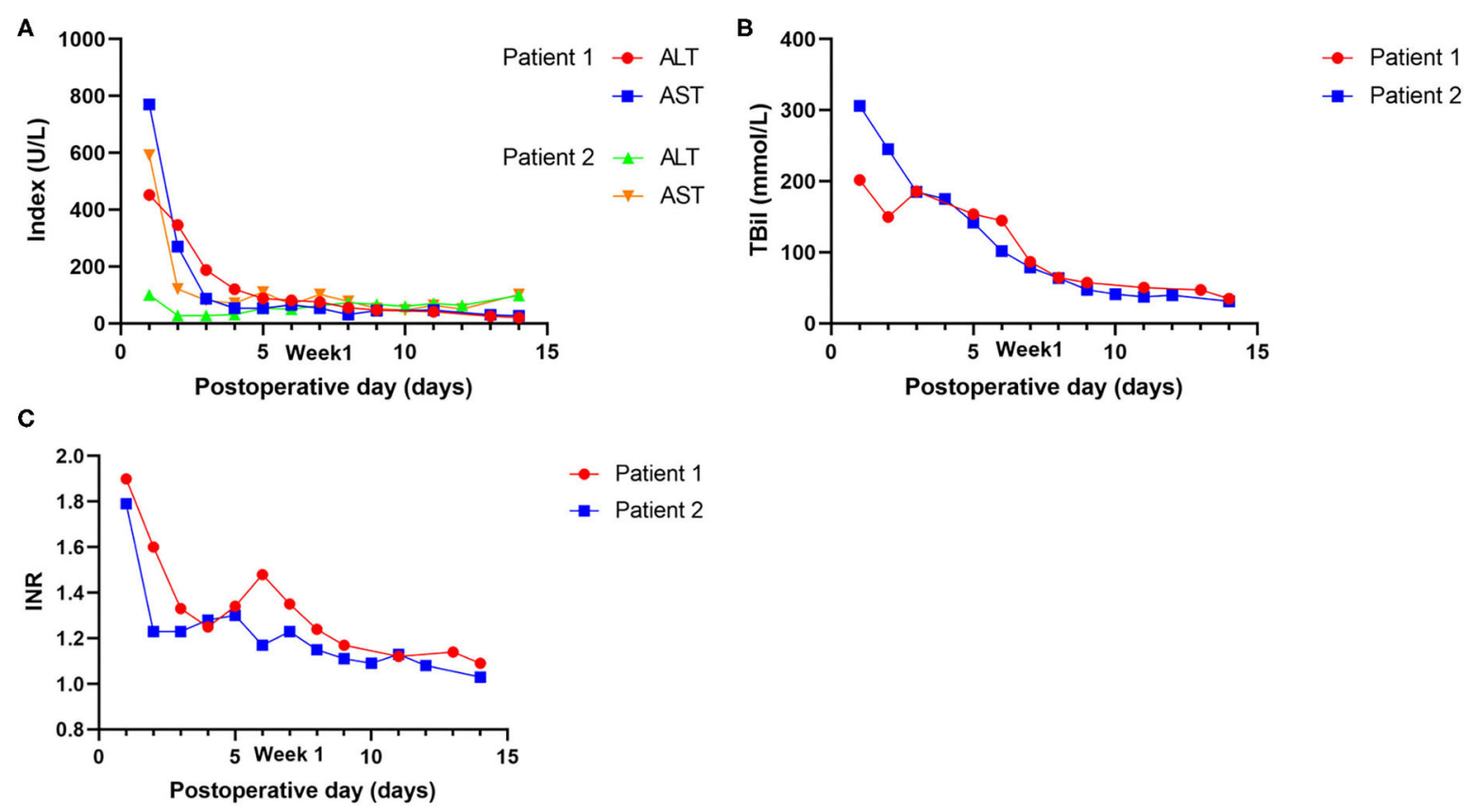

FIGURE 3 | Postoperative outcomes of laboratory tests of the recipients. (A) Changes in transaminases (AST and ALT). (B) Changes in Tbil. (C) Changes in INR. 
In conclusion, our results demonstrate that continuous NMP without recooling is efficacious and safe for LT with extended criteria donor livers.

\section{DATA AVAILABILITY STATEMENT}

The original contributions presented in the study are included in the article/supplementary material, further inquiries can be directed to the corresponding author/s.

\section{ETHICS STATEMENT}

The studies involving human participants were reviewed and approved by Institutional Ethics Committee for Clinical Research and Animal Trials of the First Affiliated Hospital of Sun Yat-sen University. Written informed consent for participation was not required for this study in accordance with the national legislation and the institutional requirements.

\section{REFERENCES}

1. Meirelles Junior RF, Salvalaggio P, Rezende MB, Evangelista AS, Guardia $\mathrm{BD}$, et al. Liver transplantation: history, outcomes and perspectives. Einstein. (2015) 13:149-52. doi: 10.1590/S1679-45082015RW3164

2. Morris PJ. Transplantation-a medical miracle of the 20th century. N Engl J Med. (2004) 351:2678-80. doi: 10.1056/NEJMp048256

3. Schlegel A, Dutkowski P: Impact of machine perfusion on biliary complications after liver transplantation. Int $J$ Mol Sci. (2018) 19:E3567. doi: 10.3390/ijms19113567

4. di Francesco F, Pagano D, Martucci G, Gruttadauria S: Normothermic machine perfusion using an air/oxygen mixer for reconditioning a liver from a marginal brain death donor. Artif Organs. (2017) 41:E668. doi: 10.1111/aor.12964

5. Houben P, Manzini G, Kremer M, Arend J, Berlakovich GA, Klar E, et al. Graft rinse prior to reperfusion in liver transplantation: literature review and online survey within the Eurotransplant community. Transplant Int. (2015) 28:1291-8. doi: $10.1111 /$ tri.12631

6. Ju W CZ, Zhao Q, Zhang Y, Huang C, Wang L, Zhu C, et al. Non-re-cooling implantation of marginal liver graft after machine perfusion: report of a case. Ann Transl Med. (2020) 8:1465. doi: 10.21037/atm-20-2774

7. Briceno J, Marchal T, Padillo J, Solorzano G, Pera C. Influence of marginal donors on liver preservation injury. Transplantation. (2002) 74:5226. doi: 10.1097/00007890-200208270-00015

8. di Francesco F, Pagano D, Martucci G, Cintorino D, Gruttadauria S. Normothermic machine perfusion in liver transplantation: feasibility and promise of avoiding recooling before engrafting. Liver Transplantation. (2019) 25:1113-7. doi: 10.1002/lt.25473

9. Durand F, Renz JF, Alkofer B, Burra P, Clavien PA, Porte RJ, et al. Report of the Paris consensus meeting on expanded criteria donors in liver transplantation. Liver Transplantation. (2008) 14:1694-707. doi: 10.1002/lt.21668

\section{AUTHOR CONTRIBUTIONS}

WJ and MC: conceptualization. ZG and XHe: methodology. $\mathrm{ZC}, \mathrm{XHo}, \mathrm{TW}$, and SH: writing-original draft. YM, CH, YG, and QZ: data collection. MC: writing-review and editing. WJ: supervision. XHe: project administration. WJ, MC, and XHe: funding acquisition. All authors contributed to the article and approved the submitted version.

\section{FUNDING}

This work was supported by the National Natural Science Foundation of China (81401324 and 81770410), Guangdong Basic and Applied Basic Research Foundation (2020A1515011557, 2020A1515010903), Science and Technology Planning Project of Guangdong Province (2016A020215048), Guangdong Provincial Key Laboratory of Organ Donation and Transplant Immunology (2020B1212060026), and Guangdong Provincial International Cooperation Base of Science and Technology (Organ Transplantation) (2015B050501002), China.

10. Maring JK, Klompmaker IJ, Zwaveling JH, Kranenburg K, Ten Vergert EM, Slooff MJ. Poor initial graft function after orthotopic liver transplantation: can it be predicted and does it affect outcome? An analysis of 125 adult primary transplantations. Clin Transplantation. (1997) 11(5 Pt 1):373-9.

11. Vodkin I, Kuo A. Extended criteria donors in liver transplantation. Clinics Liver Dis. (2017) 21:289-301. doi: 10.1016/j.cld.201 6.12 .004

12. Tchilikidi KY. Liver graft preservation methods during cold ischemia phase and normothermic machine perfusion. World J Gastrointestinal Surg. (2019) 11:126-42. doi: 10.4240/wjgs.v11.i3.126

13. Ghinolfi D, Dondossola D, Rreka E, Lonati C, Pezzati D, Cacciatoinsilla A, et al. Sequential use of normothermic regional and ex situ machine perfusion in donation after circulatory death liver transplant. Liver Transplantation. (2020) 27:385-402. doi: 10.1002/lt.25899

14. van Leeuwen $\mathrm{OB}$, de Vries Y, Fujiyoshi M, Nijsten MWN, Ubbink R, Pelgrim GJ, et al. Transplantation of high-risk donor livers after ex situ resuscitation and assessment using combined hypo- and normothermic machine perfusion: a prospective clinical trial. Annals Surg. (2019) 270:90614. doi: 10.1097/SLA.0000000000003540

Conflict of Interest: The authors declare that the research was conducted in the absence of any commercial or financial relationships that could be construed as a potential conflict of interest.

Copyright (C) 2021 Chen, Hong, Huang, Wang, Ma, Guo, Huang, Zhao, Guo, He, Ju and Chen. This is an open-access article distributed under the terms of the Creative Commons Attribution License (CC BY). The use, distribution or reproduction in other forums is permitted, provided the original author(s) and the copyright owner(s) are credited and that the original publication in this journal is cited, in accordance with accepted academic practice. No use, distribution or reproduction is permitted which does not comply with these terms. 